JOURNAL OF MECHANICS OF MATERIALS AND STRUCTURES

Vol. 3, No. 1, 2008

\title{
FLEXURE OF BEAMS WITH AN INTERLAYER - SYMMETRIC BEAMS WITH ORTHOTROPIC ADHERENDS
}

\author{
K. S. Alfredsson, T. A. Bogetti, L. A. Carlsson, J. W. Gillespie, Jr. And A. Yiournas
}

\begin{abstract}
Analysis of the three-point flexure loading of a symmetric beam configuration consisting of two identical adherends joined by a flexible layer is presented. A closed-form solution is presented in the form of a layer-wise approach, and shows excellent agreement with finite element results. Particular emphasis is given to the global beam compliance. The results show that a thin, shear deformable layer may effectively decouple the responses of the two beams. Furthermore, in contrast to ordinary beams, the presence of an overhang reduces the beam compliance. These effects are quantified and discussed. Experiments on aluminum adherends joined by a rubber layer were conducted over a range of overhang lengths in a three-point flexure fixture, and very good agreement between measured and predicted beam compliance was noted.
\end{abstract}

\section{Introduction}

Sandwich structures are commonly designed in an effort to mimic the I-beam concept where the web supports shear loads and the flanges bending loads. In certain structural applications, however, it may be desirable to utilize a highly shear deformable layer sandwiched between more rigid adherends, such as in structures utilizing shear thickening fluids to control the vibration response; see [Fischer et al. 2006], and in mechanical shielding of brittle layers; see for example [Mahdi et al. 2003].

The design of such structures is greatly benefited by an improved understanding of the role of the thickness and stiffness of the rubber layer in its ability to interact with the upper and lower layers of the structure. Most analysis of such structures to date is based on finite element analysis [Davila and Chen 2000; Mahdi and Gillespie 2004], because it is widely recognized that the large local shear deformation of the layer prohibits the use of ordinary beam and plate theories [Timoshenko and Woinowsky-Kreiger 1959; Gere and Timoshenko 1984] and first order shear deformation theory [Whitney 1973]. Sandwich beams and panels with shear-deformable compliant core materials have been considered by numerous researchers since the pioneering work of Reissner [1947, 1948]; see, for example, [Zenkert 1997]. Such analysis, however, is applicable to structures with thin faces over a thick core, while the present work considers thick adherends over a thin flexible layer.

In this paper we will present a layer-wise analytical beam approach to the mechanics of symmetric beam configurations with a flexible interlayer. Use of the simplest possible formulation that can capture the global bending behavior allows us to obtain a closed form solution. The approach shares several

Keywords: flexure, interlayer, shear deformation.

The authors would like to acknowledge funding from the Army Research Laboratory (Cooperative Agreement \# DAAD1901-2-0005) and the Composite Materials Center of Excellence program at the University of Delaware Center for Composite Materials. 


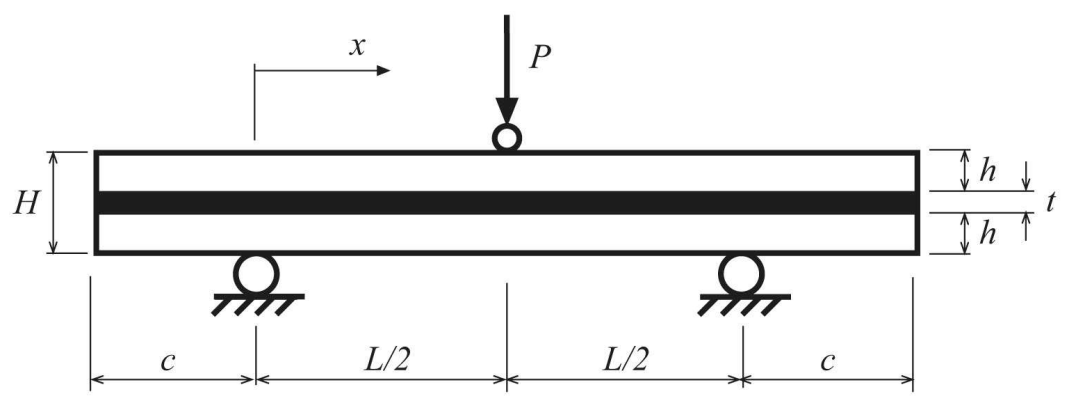

Figure 1. Three-point flexure loading of a layered beam with symmetric overhangs.

elements with the layer-wise approach developed for sandwich beams with a flexible core by Frostig and Baruch [1990], although they considered a thick core between thin faces and addressed local indentation at load introduction and support regions, while the main focus of our approach is on the global beam response and shear transfer effects. Alfredsson [2004] examined adhesively bonded end-notch flexure (ENF) fracture test specimens, but his analysis is valid only for infinitely thin layers. The analysis will be compared to finite element (FE) results and to experimental compliance data determined for beams with a rubber layer tested in three-point flexure loading.

\section{Problem description}

A multilayer symmetric beam structure consisting of two stiff and thick orthotropic adherends separated by a flexible isotropic interlayer loaded in three-point flexure is considered (Figure 1). In this initial study, the adherends are assumed to be of identical thickness. The solution is simplified to isotropic adherends to facilitate correlation with the experimental compliance data. A major objective here is to determine the global beam deflection. As will be shown, the overhang length $c$ influences the compliance, and the role of this parameter will be examined.

For the symmetrical beam configuration considered (Figure 1), the loading may be divided into symmetric and antisymmetric parts (Figure 2), and each solution is superimposed to obtain the solution to the original problem. It is recognized that the symmetric part of the loading produces extensional (tensile or compressive) deformation of the interlayer, while the antisymmetric part produces shear deformation of the interlayer and bending of the layered beam. The symmetric part of the loading will not contribute to the global bending of the layered beam, and for this reason, only the antisymmetric part of the loading is considered here.

\section{Derivation of governing equations}

The adherends are considered to be Euler-Bernoulli beams. Considering only the antisymmetric part of the loading means that the resulting displacement field is also antisymmetric. Hence, the two adherends will have equal deflection, which is denoted $w$. The longitudinal displacements of the midplanes of the adherends are of equal magnitude, but of opposite sign. The displacement in the $x$ direction (Figure 1) of the midplane of the lower adherend is denoted by $u$; see Figure 3. 


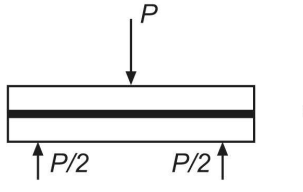

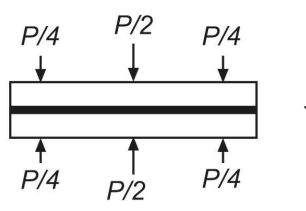

SYMMETRIC

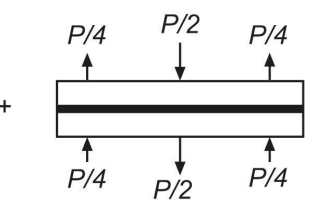

ANTISYMMETRIC

Figure 2. Superposition of symmetric and antisymmetric loadings.

The deformation of the interlayer is defined as the relative displacements of the lower and upper adherend/interlayer interfaces. According to classical beam theory, the cross sections of the adherends are assumed to undergo translation and rotation only. Hence, the deformation of the interlayer is defined with respect to the midplane of each adherend. As shown in Figure 3, both the axial displacement of each adherend, $u$, and the rotation of the beam, $w^{\prime}$, contribute to the total shear deformation $v$ of the flexible layer

$$
v(x)=2 u(x)+(h+t) w^{\prime}(x)
$$

Longitudinal equilibrium of the flexible interlayer is assumed to be fulfilled by a shear stress, $\tau$, which is constant throughout the thickness of the layer; see Figure 4. This means that the longitudinal normal stress in the flexible layer is neglected, which is justified since it is much smaller than the longitudinal normal stresses in the adherends and the shear stress in the flexible layer. The antisymmetric condition implies that there are no transverse normal stresses at the symmetry line passing through the middle of the interlayer. However, at the interface between the interlayer and the adherends, a normal stress $\sigma$ may be present; see Figure 4.

Vertical equilibrium of the flexible layer requires that

$$
2 \sigma(x)=t \tau^{\prime}(x),
$$

where $t$ is the thickness of the flexible layer; see Figure 1.

The adherends transmit normal forces, $N$, bending moments, $M$, and transversal shear forces, $V$. The positive directions of these stress resultants are shown in Figure 4 for the antisymmetric loading case.
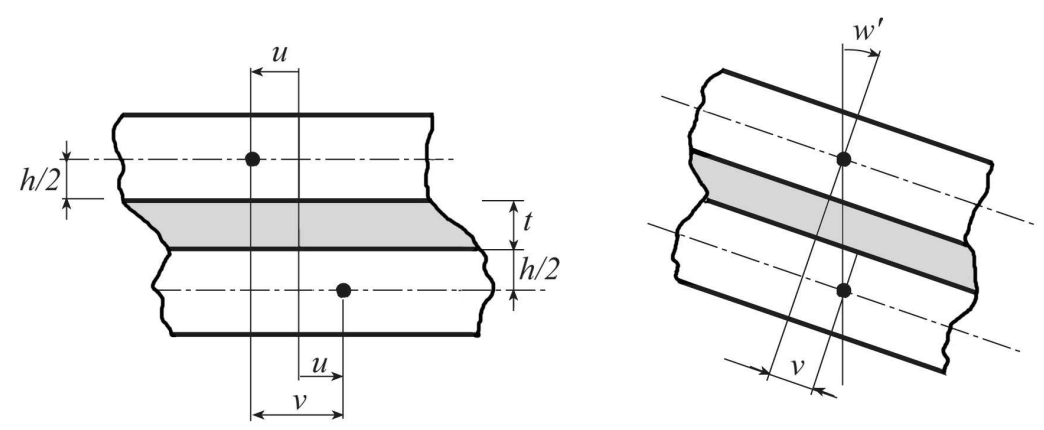

Figure 3. Shear deformation of a flexible layer due to longitudinal displacements, $u$ (left), and slope, $w^{\prime}$ (right). 


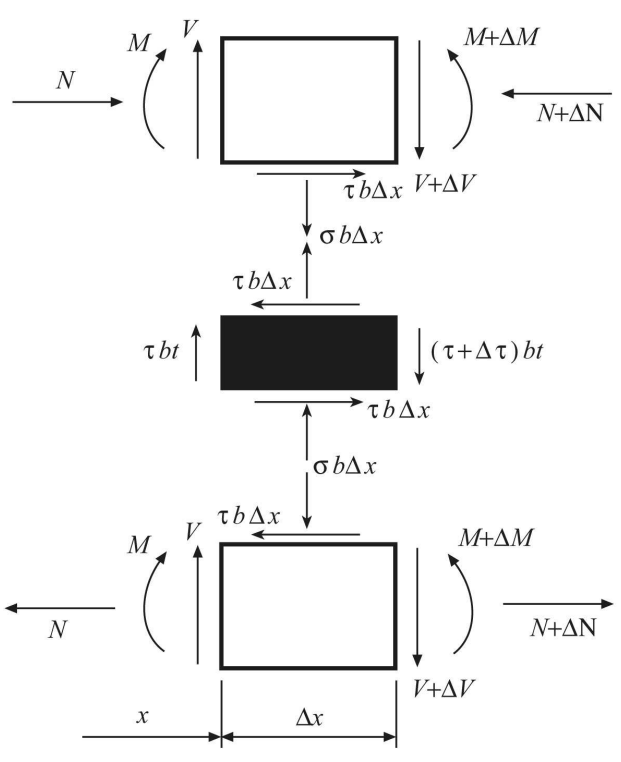

Figure 4. Positive direction of adhesive stresses and sectional loads.

Equilibrium of the adherends requires that

$$
\begin{aligned}
N^{\prime}(x) & =b \tau[v(x)], \\
V^{\prime}(x) & =-b \sigma(x), \\
V(x) & =M^{\prime}(x)+\frac{1}{2} b h \tau[v(x)],
\end{aligned}
$$

where $b$ is the specimen width and $h$ is the adherend thickness.

Assuming that the adherends deform elastically according to the Euler-Bernoulli beam theory, the normal force and the bending moment are given by

$$
\begin{aligned}
& N(x)=A u^{\prime}(x), \\
& M(x)=-D w^{\prime \prime}(x),
\end{aligned}
$$

where $A=b \int E_{1}(z) d z$ and $D=b \int E_{1}(z) z^{2}$ are the extensional and flexural stiffnesses of the adherends. $E_{1}$ is the Young's modulus in the longitudinal direction of the adherends. Furthermore, $z$ is a coordinate in the thickness direction. For isotropic adherends with a Young's modulus $E$, integration yields

$$
A=E b h \quad \text { and } \quad D=E b h^{3} / 12 .
$$

In order to define a boundary value problem which involves only the shear deformation, the longitudinal displacement and the deflection are eliminated from the governing equations. Combination of Equations (2) and (3b) yields

$$
2 V^{\prime}(x)+b t \tau^{\prime}(x)=0 .
$$


Integration yields

$$
2 V(x)+b t \tau(x)=\bar{V}
$$

where $\bar{V}$ is the total shear force transmitted by the sandwich beam; see Figure 5 . This equation can also be obtained directly from Figures 4 and 5. Substitution of Equation (3c) into Equation (5) yields the following differential equation:

$$
2 M^{\prime}(x)+b(h+t) \tau[v(x)]=\bar{V},
$$

where the shear stress in the layer, $\tau$, is a function of the shear deformation, $v$. Next, $\tau$ is substituted from Equation (3a) into Equation (6). With $N$ and $M$ expressed in terms of the displacements $u$ and $w$ according to Equation (4), the result is,

$$
2 w^{\prime \prime \prime}(x)-\frac{A}{D}(h+t) u^{\prime \prime}(x)=-\frac{\bar{V}}{D} .
$$

Differentiating Equation (1) twice and combining the result with Equation (7) yields a relation between the second derivatives of $u$ and $v$,

$$
\left[2+\frac{A}{2 D}(h+t)^{2}\right] u^{\prime \prime}(x)=v^{\prime \prime}(x)+\frac{\bar{V}}{2 D}(h+t)
$$

Substitution of the second derivative of $u$ from Equation (8) into the equation obtained by combining Equations (3a) and (4a), the following differential equation relating shear deformation and shear stress is found:

$$
v^{\prime \prime}(x)=\frac{b}{A}\left[2+\frac{A}{2 D}(h+t)^{2}\right]\{\tau[v(x)]-\bar{\tau}\},
$$

where

$$
\bar{\tau}=\frac{\bar{V}}{b h} \frac{1+\frac{t}{h}}{\frac{4 D}{A h^{2}}+\left(1+\frac{t}{h}\right)^{2}}=\frac{3}{4} \frac{\bar{V}}{b h} \frac{1+\frac{t}{h}}{1+\frac{3}{2} \frac{t}{h}+\frac{3}{4}\left(\frac{t}{h}\right)^{2}} .
$$

Here, $\bar{\tau}$ is the constant shear stress in the interlayer predicted by ordinary beam theory, [Gere and Timoshenko 1984]. For a small deformation of the interlayer, a linear constitutive relation applies. Thus, for the shear deformation,

$$
\tau(x)=k v(x), \quad k=\frac{G}{t},
$$

where $G$ is the shear modulus of the layer. Due to the assumed antisymmetric loading (Figures 2, and 4), no transverse deformation of the interlayer will take place since the transverse stress in the interlayer

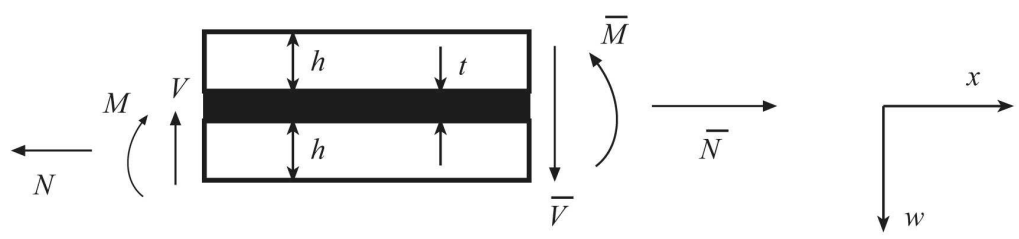

Figure 5. Sectional loads for entire beam sections; $\bar{N}, \bar{M}$, and $\bar{V}$, and for adherends $N$, $M$, and $V$. 
varies linearly with a zero average. Substitution of Equation (11) into Equation (9) yields a linear differential equation of second order:

$$
v^{\prime \prime}(x)-\kappa^{2} v(x)=-\kappa^{2} \bar{\tau} / k,
$$

with

$$
\kappa=\sqrt{\left[2+\frac{A}{2 D}(h+t)^{2}\right] \frac{b G}{A t}}=\sqrt{\frac{8 G}{E h t}\left[1+\frac{3}{2} \frac{t}{h}+\frac{3}{4}\left(\frac{t}{h}\right)^{2}\right]} .
$$

As will be further discussed in this paper, $\kappa$ constitutes an inverse length scale of the solution. The general solution to Equation (12) is

$$
v(x)=A_{1} e^{\kappa x}+A_{2} e^{-\kappa x}+\frac{\bar{\tau}}{k},
$$

where $A_{1}$ and $A_{2}$ are integration constants determined by boundary conditions on $v$ or $v^{\prime}$.

The following relation, obtained from Equation (1) and Equation (4), can be used to transform boundary conditions on $N$ and $M$ into boundary conditions on $v^{\prime}$,

$$
v^{\prime}(x)=\frac{2}{A} N(x)-\frac{h+t}{D} M(x) .
$$

From this equation it also follows that not only $v$ but also $v^{\prime}$ are continuous when no concentrated longitudinal forces or moments are applied to the adherends. Specific solutions will be discussed for beams without and with overhang.

\section{Beams without overhang}

Consider first a beam without overhang, that is, $c=0$ in Figure 1. Due to symmetry, only one half of the specimen, here the left part, $0 \leq x \leq L / 2$, must be analyzed. At the left end, $x=0$, the adherends are unloaded, with $N(0)=0$ and $M(0)=0$. From Equation (15), the boundary conditions on $N$ and $M$ can be translated to a boundary condition on $v^{\prime}$. Since the shear stress in the flexible layer is antisymmetric with respect to the loading point, $x=L / 2$, the shear deformation is zero at $x=L / 2$. Thus, the boundary conditions are:

$$
v^{\prime}(0)=0, \quad v(L / 2)=0 .
$$

Subjecting the general solution, Equation (14), to these conditions, the shear stress takes the form

$$
\tau(x)=\bar{\tau}\left[1-\frac{\cosh (\kappa x)}{\cosh (\kappa L / 2)}\right], \quad 0 \leq x \leq L / 2,
$$

where $\bar{\tau}$ is given by Equation (10) with $\bar{V}=P / 2$. The distribution of interlayer shear stress along the beam is shown in Figure 6 for three span lengths. The results are normalized by $\bar{\tau}$. For long beams $(L=2.0 \mathrm{~m})$ the shear stress is close to the ordinary beam theory result $(\bar{\tau})$ everywhere except near the loading point where a sign change takes place. This can also be deduced from Equation (17); that is, for large values of $L$ the second term inside the parentheses is negligible except when $x$ is close to $L / 2$. Hence, with respect to the shear stress distribution in the interlayer, the present theory degenerates into ordinary beam theory for large span lengths. The shear stress decreases with decreasing span length. 


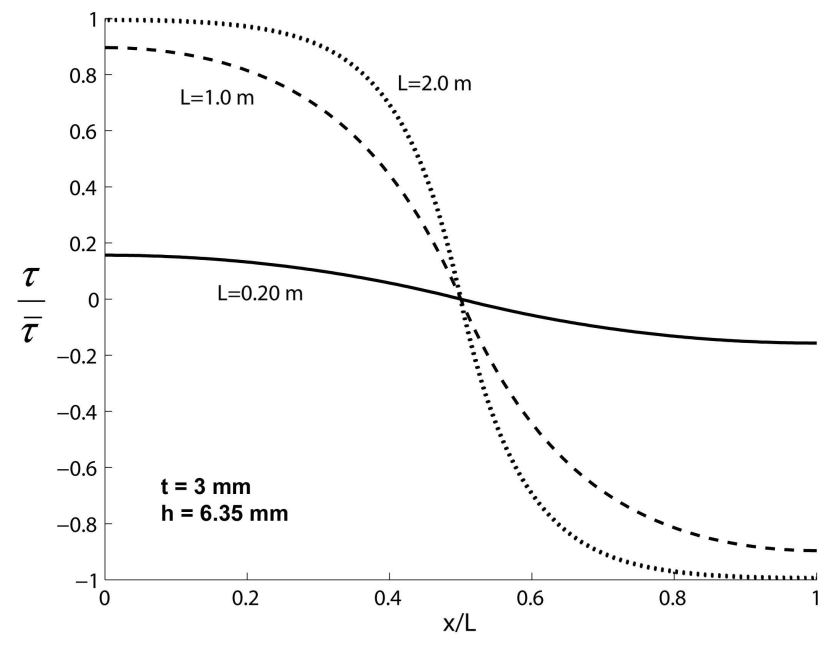

Figure 6. Shear stress distribution along the beam for three span lengths, $L=.2,1$ and $2 \mathrm{~m}, E=70 \mathrm{GPa}, G=3.1 \mathrm{MPa}, h=6.35 \mathrm{~mm}, t=3 \mathrm{~mm}$. The shear stress is normalized by the value obtained from ordinary beam theory.

For a short span length $(L=0.2 \mathrm{~m})$, the maximum shear stress is only about $20 \%$ of the ordinary beam theory value.

The normal force in the adherends is determined by integration of Equation (3a),

$$
N(x)=b \int_{0}^{x} \tau[v(\tilde{x})] d \tilde{x}=\frac{b \bar{\tau}}{\kappa}\left[\kappa x-\frac{\sinh (\kappa x)}{\cosh (\kappa L / 2)}\right] .
$$

The bending moment in the adherends is determined from the equilibrium of a free body element of the beam cut at a distance $x$ from the left end:

$$
M(x)=\frac{1}{4} P x-\frac{1}{2}(h+t) N(x)=\frac{b h \bar{\tau}}{2 \kappa(1+t / h)}\left[\frac{4 D}{A h^{2}} \kappa x+\left(1+\frac{t}{h}\right)^{2} \frac{\sinh (\kappa x)}{\cosh (\kappa L / 2)}\right],
$$

where $P=2 \bar{V}$ and Equations (10) and (18) have been used to obtain the final expression for $M(x)$ above. Notice that for isotropic adherends, $A h^{2} /(12 D)=1$.

The deflection, $w(x)$, is determined by integrating Equation (4b) and using the boundary conditions, $w(0)=0$ and $w^{\prime}(L / 2)=0$. The beam compliance, $C=w(L / 2) / P$ takes the form,

$$
\frac{C}{C_{b t}}=1+\frac{A h^{2}}{12 D}\left(1+\frac{t}{h}\right)^{2}\left[\frac{36}{\kappa^{2} L^{2}}-\frac{72}{\kappa^{3} L^{3}} \tanh \left(\frac{\kappa L}{2}\right)\right],
$$

where $C_{b t}$ is the compliance according to ordinary beam theory,

$$
C_{b t}=\frac{L^{3}}{96 D+24 A(h+t)^{2}}=\frac{L^{3}}{32 E b h^{3}}\left[1+\frac{3}{2} \frac{t}{h}+\frac{3}{4}\left(\frac{t}{h}\right)^{2}\right]^{-1} .
$$

It is seen from Equation (19) that for large values of $L$ we have $C \approx C_{b t}$, which implies that with respect to the compliance, the present theory degenerates into ordinary beam theory for large span lengths. 
In an attempt to clarify the implications of Equation (19), two definitions referring to the effects of introducing a flexible layer, thickness effect and flexibility effect, are introduced. The thickness effect refers to the increase in flexural stiffness (decrease of compliance) due to the thickness of the flexible layer. If only the thickness effect is accounted for, the compliance equals $C_{b t}$ (Equation (20)), the compliance predicted by ordinary beam theory.

The flexibility effect refers to the decoupling of the two adherends due to shear deformation of the layer, but disregards the increased thickness. To isolate this effect, it is assumed that $t / h \ll 1$, and Equation (19) becomes

$$
\frac{C_{f}}{C_{0}}=1+\frac{A h^{2}}{12 D}\left[\frac{36}{\bar{\kappa}^{2} L^{2}}-\frac{72}{\bar{\kappa}^{3} L^{3}} \tanh \left(\frac{\bar{\kappa} L}{2}\right)\right], \quad \frac{t}{h} \ll 1,
$$

where

$$
\begin{aligned}
\bar{\kappa} & =\sqrt{\left[2+\frac{A h^{2}}{2 D}\right] \frac{b G}{A t}}=\sqrt{\frac{8 G}{E h t}}, \\
C_{0} & =\frac{L^{3}}{96 D+24 A h^{2}}=\frac{L^{3}}{32 E b h^{3}},
\end{aligned}
$$

in which $C_{0}$ is the compliance when the two outer beams are fully coupled by an interlayer of zero thickness.

Figure 7 illustrates the influences of the two effects on the beam compliance, here normalized by $C_{0}$ (Equation (22b)). The dotted curve shows the monotonic decrease of the compliance according to ordinary beam theory, $C_{b t}$ in Equation (20), due to the increased interlayer thickness. The dashed curve shows the monotonic increase of $C_{f}$ (Equation (21)) due to the increase of the flexibility, $k^{-1}=t / G$, with increasing interlayer thickness. The solid curve represents the actual compliance, $C$, predicted by

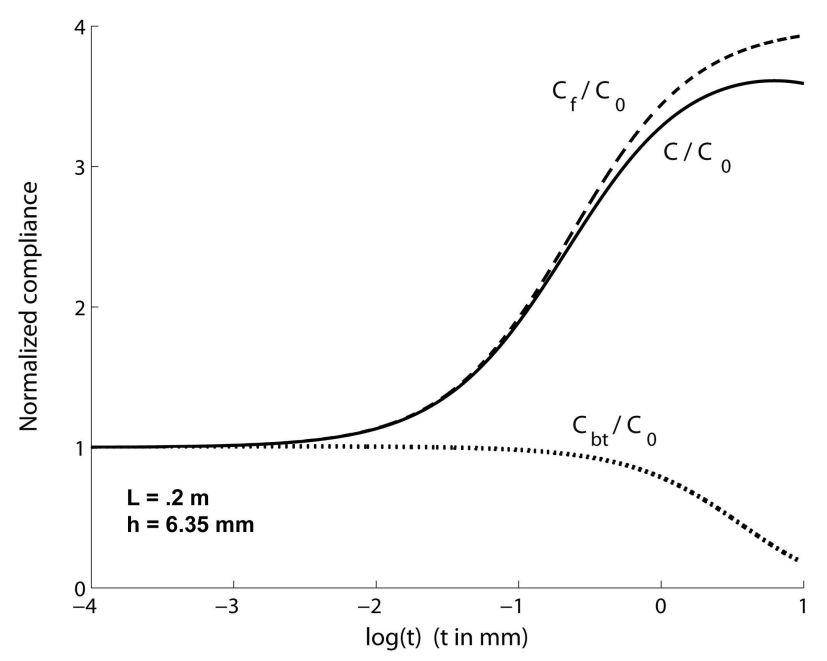

Figure 7. Compliance as a function of layer thickness. $E=70 \mathrm{GPa}, G=3.1 \mathrm{MPa}$, $h=6.35 \mathrm{~mm}, L=0.2 \mathrm{~m}$. 
Equation (19). It is seen that the actual compliance $C$ is bounded by $C_{b t}$ and $C_{f}$. That is, when only the thickness effect is accounted for (ordinary beam theory) the compliance is underestimated, and when only the flexibility effect is accounted for, the compliance is overestimated. It can be shown mathematically that this is always the case, that is, $C_{b t} \leq C \leq C_{f}$.

Figure 8 shows the compliance calculated from Equation (19), normalized by $C_{0}$, as a function of layer thickness for span lengths of $.2,1$, and $2 \mathrm{~m}$. For the shortest span $(L=.2 \mathrm{~m})$, the compliance increases with increasing layer thickness over the entire interval studied. This means that for large layer thicknesses, the two adherends are practically decoupled and bend independently of one another, that is, the flexibility effect dominates. Such beams actually have two neutral planes with each plane on the geometrical center of each adherend. For a beam with a large span $(L=2.0 \mathrm{~m})$, the compliance decreases with layer thickness, that is, the thickness effect dominates the behavior. For the intermediate span length $(L=1.0 \mathrm{~m})$, the compliance increases with layer thickness up to a critical value of $t_{c r} \approx 3.5 \mathrm{~mm}$. Below this value the flexibility effect dominates. For layer thicknesses above $3.5 \mathrm{~mm}$, the thickness effect dominates and the compliance decreases with increasing layer thickness.

From the results shown in Figure 8, it becomes clear that for a specified choice of adherends and a given interlayer material, there exists a critical span length, $L_{c r}$, above which the addition of a thin flexible layer will not decouple the response - that is, the compliance will not increase when a thin flexible interlayer is added. The critical span length is found by making $C$ stationary with respect to $t$ for $t \rightarrow 0$, which yields,

$$
L_{c r}=\sqrt{\frac{3 A h}{b G}}=h \sqrt{\frac{3 E}{G}} .
$$

For the present geometry the critical span length is $1.65 \mathrm{~m}$; see Figure 8 . This equation should be useful for the design of structures containing flexible interlayers.

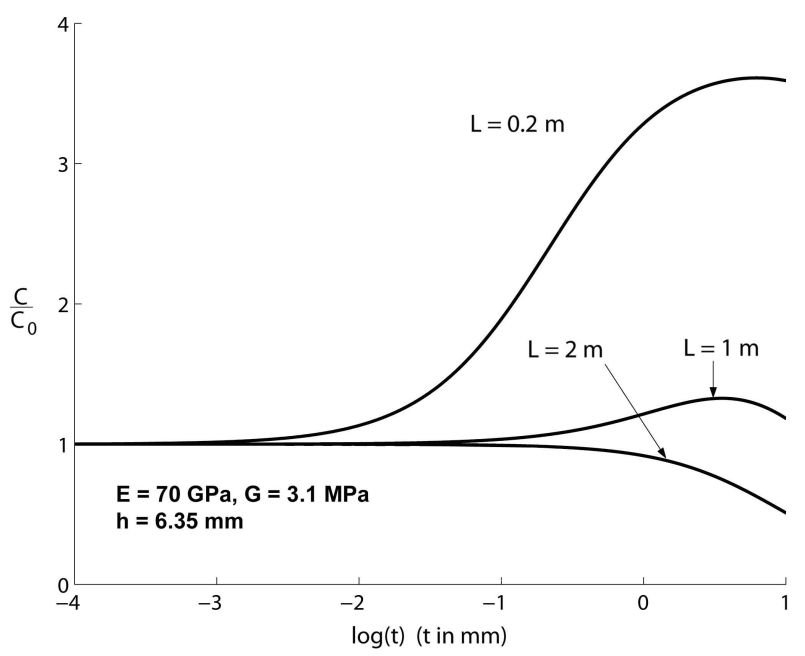

Figure 8. Compliance as a function of layer thickness for span lengths of $.2,1$ and $2 \mathrm{~m}$ $E=70 \mathrm{GPa}, G=3.1 \mathrm{MPa}, h=6.35 \mathrm{~mm}$. 
Equation (21) can also be used to examine the behavior for beams with a very thin $(t / h \ll 1)$ layer. If such a layer is very stiff, $G \rightarrow \infty$, the compliance approaches that of a homogeneous beam, and $C \rightarrow C_{0}$. On the other hand, if the thin layer is very flexible, $G \rightarrow 0, \bar{\kappa} \rightarrow 0$, then the compliance $C \rightarrow 4 C_{0}$ for the case of isotropic adherends. This case effectively corresponds to one beam sliding on top of the other. The beams may be said to be fully decoupled if the compliance is more that $99 \%$ of the asymptotic value, $C=4 C_{0}$. The other extreme, fully coupled, may be defined when the compliance exceeds the lower limit, $C=C_{0}$, by less than $1 \%$. These conditions can be quantified using Equation (21). The degree of decoupling is characterized by introduction of the following dimensionless decoupling parameter,

$$
\beta=\frac{8}{\bar{\kappa}^{2} L^{2}}=\frac{E}{G}\left(\frac{h}{L}\right)^{2} \frac{t}{h}
$$

The variation of compliance with $\beta$ is shown in Figure 9. The curve can be used to assess the level of decoupling by calculating $\beta$ for any particular beam configuration. The curve also shows that for a very thin layer it is required that $\beta<0.0023$ to achieve full coupling of the two beams $\left(C / C_{0}<1.01\right)$. On the other hand, $\beta>59$ gives full decoupling of the two beams $\left(C /\left(4 C_{0}\right)>0.99\right)$.

\section{Beams with overhang}

Consider a beam with symmetric overhang, that is, $c>0$ in Figure 1. Due to symmetry, only the left part, $-c \leq x \leq L / 2$, is studied. The total shear force is discontinuous at the supports:

$$
\bar{V}(x)= \begin{cases}0, & \text { for }-c \leq x<0 \\ P / 2, & \text { for } 0<x<L / 2 .\end{cases}
$$

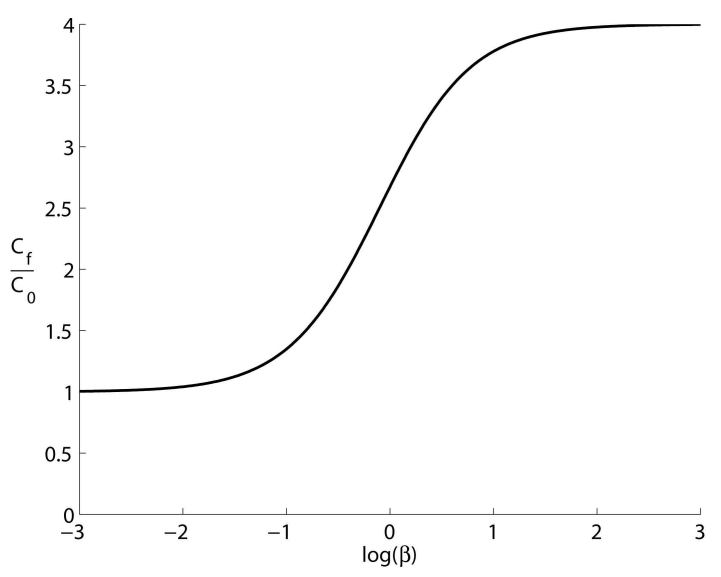

Figure 9. Compliance as a function of decoupling parameter, $\beta$, for thin interlayers, $t / h \ll 1$. 
According to Equation (14), and the procedure outlined for the $c=0$ case, this implies that the general solution for the shear deformation of the interlayer is given by

$$
v(x)= \begin{cases}\left(A_{1} e^{\kappa x}+A_{2} e^{-\kappa x}\right) \frac{\bar{\tau}}{k}, & \text { for }-c \leq x \leq 0 \\ \left(A_{3} e^{\kappa x}+A_{4} e^{-\kappa x}+1\right) \frac{\bar{\tau}}{k}, & \text { for } 0 \leq x \leq L / 2,\end{cases}
$$

where $\bar{\tau}$ is given by Equation (10) with $\bar{V}=P / 2$. The absence of a constant term for the overhang portion in Equation (23) reflects the fact that the total shear force, $\bar{V}$, Figure 5, is zero in the overhang part of the beam. The boundary conditions at the free left end, $x=-c$, and at the midspan, $x=L / 2$, are, similar to Equation (16),

$$
\begin{gathered}
v^{\prime}(-c)=0, \\
v(L / 2)=0 .
\end{gathered}
$$

Moreover, both $v$ and $v^{\prime}$ must be continuous at the support, $x=0$. With these four conditions, the integration constants become

$$
\begin{aligned}
& A_{1}=\frac{1}{2} \frac{e^{\kappa L / 2}+e^{-\kappa L / 2}-2}{e^{\kappa L / 2}+e^{-\kappa(4 c+L) / 2}}, \\
& A_{2}=A_{1} e^{-2 \kappa c}=\frac{1}{2} \frac{e^{\kappa(L-4 c) / 2}+e^{-\kappa(4 c+L) / 2}-2 e^{-2 \kappa c}}{e^{\kappa L / 2}+e^{-\kappa(2 c+L / 2)}}, \\
& A_{3}=A_{1}-\frac{1}{2}=\frac{1}{2} \frac{e^{-\kappa L / 2}-e^{-\kappa(4 c+L) / 2}-2}{e^{\kappa L / 2}+e^{-\kappa(4 c+L) / 2}}, \\
& A_{4}=A_{2}-\frac{1}{2}=\frac{1}{2} \frac{e^{\kappa(L-4 c) / 2}-e^{\kappa L / 2}-2 e^{-2 \kappa c}}{e^{\kappa L / 2}+e^{-\kappa(4 c+L) / 2}} .
\end{aligned}
$$

The longitudinal force, $N$ (Figure 3), is obtained by integration of Equation (3a),

$$
N(x)=b \int_{-c}^{x} \tau[v(\tilde{x})] d \tilde{x} .
$$

The bending moment in the adherends is determined from equilibrium:

$$
M(x)= \begin{cases}-\frac{1}{2}(h+t) N(x), & \text { for }-c \leq x \leq 0 \\ \frac{1}{4} P x-\frac{1}{2}(h+t) N(x), & \text { for } 0 \leq x \leq L / 2 .\end{cases}
$$

With the bending moment determined, the deflection, $w(x)$, is determined by integration of Equation (4b). With the boundary conditions, $w(0)=0$ and $w^{\prime}(L / 2)=0$, the compliance, $C_{o h}=w(L / 2) / P$, takes the form,

$$
\frac{C_{o h}}{C_{b t}}=1+\left(\frac{A h^{2}}{12 D}\right) \frac{36}{\kappa^{3} L^{3}}\left(1+\frac{t}{h}\right)^{2}\left[A_{3}\left\{e^{\kappa L / 2}(2-\kappa L)-2\right\}+A_{4}\left\{2-e^{-\kappa L / 2}(2+\kappa L)\right\}\right],
$$


where $A_{3}, A_{4}$ and $C_{b t}$ are given in Equations (25c), (25d), and (20). For zero overhang length, $c=0$, it can be shown that $C_{o h}$ degenerates into $C$ according to Equation (19). When the overhang is infinitely long, the limit compliance is given by

$$
\frac{C_{o h}}{C_{b t}}=1+\left(\frac{A h^{2}}{12 D}\right) \frac{36}{\kappa^{3} L^{3}}\left(1+\frac{t}{h}\right)^{2}\left[\kappa L-3-e^{-\kappa L}+4 e^{-\kappa L / 2}\right] .
$$

It will be demonstrated below that even though $C_{\infty}$ is an asymptotic value for infinitely long overhangs, it is also a very good approximation for moderate values of the overhang. Figure 10 shows the compliance for beams with overhang normalized by the limiting value of the compliance. The data are plotted against the dimensionless parameter $\kappa c$. It is noted that the overhang does not influence the compliance very much at longer spans. Figure 10 also shows that for $\kappa c>3$ the limit value of the compliance, $C_{\infty}$, is a very good approximation, regardless of the span length. Hence, from Equation (13) it follows that compliance, $C_{o h}$, is very close to the limiting value of the compliance, $C_{\infty}$, if the overhang length exceeds the limiting overhang length, $c_{\infty}$,

$$
c_{\infty}=3 \sqrt{\frac{A t}{b G}\left[2+\frac{A}{2 D}(h+t)^{2}\right]^{-1}}=3 \sqrt{\frac{E h t}{8 G}\left[1+\frac{3}{2} \frac{t}{h}+\frac{3}{4}\left(\frac{t}{h}\right)^{2}\right]^{-1}} .
$$

For isotropic adherends and a thin flexible layer $(t / h \ll 1)$, the limiting overhang is given by

$$
\bar{c}_{\infty}=3 \sqrt{\frac{E h t}{8 G}} .
$$

This expression resembles the one for the decay length of end effects for composites according to St. Venant's principle ([Horgan 1972; Choi and Horgan 1977; Horgan and Knowles 1983; Bogetti et al. 1988]) in the sense that the square root of the ratio of the Young's and shear moduli governs the length scale.

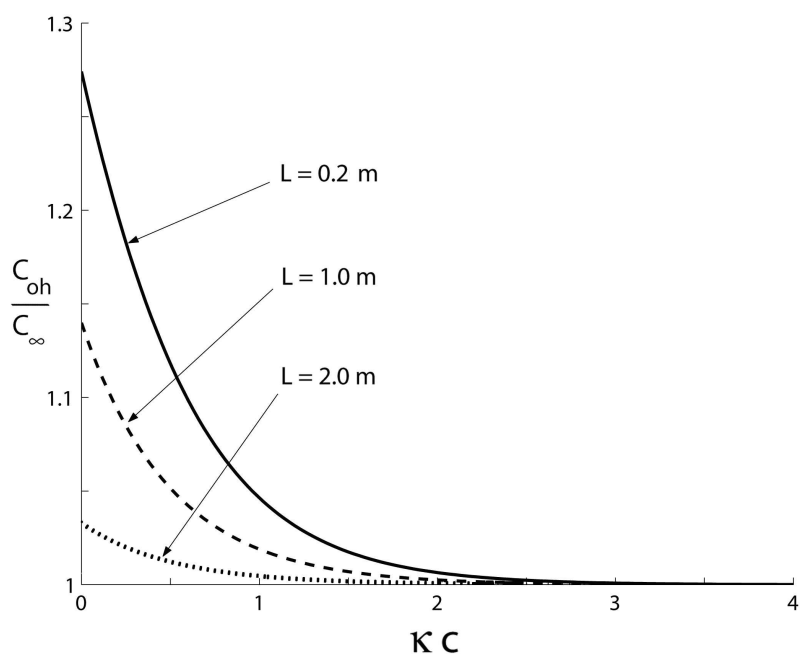

Figure 10. Compliance as a function of overhang length $(\kappa c)$, for span lengths, of .2, 1 and $2 \mathrm{~m}$. $E=70 \mathrm{GPa}, G=3.1 \mathrm{MPa}, h=6.35 \mathrm{~mm}, t=3 \mathrm{~mm}$. 


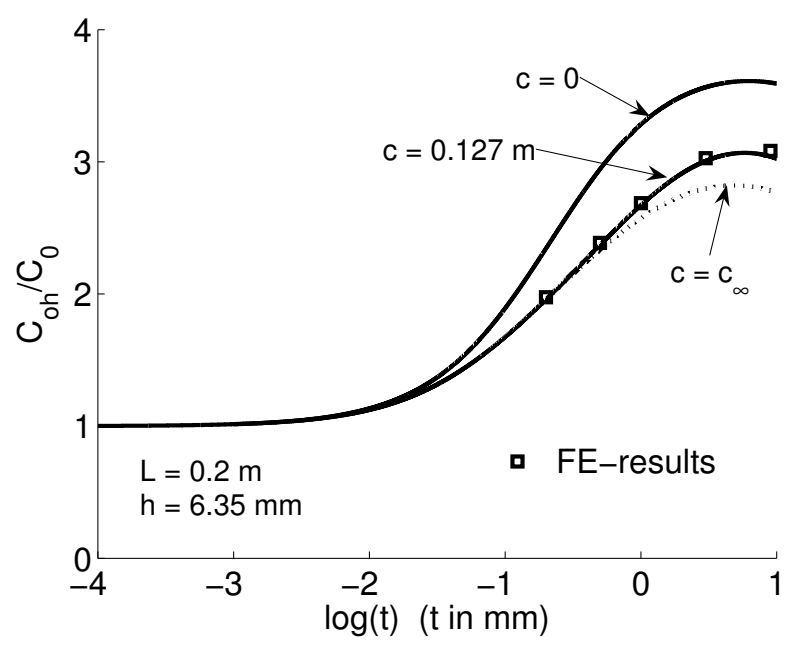

Figure 11. Compliance as a function of layer thickness for overhang lengths, $c$, of 0 , $.127 \mathrm{~m}$, and $c_{\infty} . E=70 \mathrm{GPa}, G=3.1 \mathrm{MPa}, h=6.35 \mathrm{~mm}$, and $L=0.2 \mathrm{~m}$. The open squares represent results from FE analysis with Poisson's ratio $v=0.4999$ for the rubber and $v=0.33$ for the aluminum.

Figure 11 shows the compliance $C_{o h} / C_{0}$ as a function of the layer thickness for overhang lengths $c=0, .127 \mathrm{~m}$, and $c_{\infty}$. The reduction of the compliance by the overhang segments is quite substantial for thick layers. A comparison with a two-dimensional continuum finite element model is also made in Figure 11 for the case of $c=.127 \mathrm{~m}$. The adherends are assumed to deform under plane stress in order to conform to the beam theory solution. The flexible interlayer is constrained in its plane by the stiff adherends, and is thus assumed to deform under plane strain. The FE code [Mechanica 2007] is used in the FE simulations. A ninth order polynomial is used for interpolation of displacements along all element edges. Sixteen elements are used along the span. The elements in the overhang regions are of the same lengths as those inside the span. One element through the thickness of each adherend and the interlayer is used. This is sufficient due to the high degree of the interpolation polynomial. The FE results are shown as squares in Figure 11. The correspondence to the analytical solution is very good. For large interlayer thicknesses a small deviation is observed, and the FE solution gives a slightly higher compliance. This is due to compression of the interlayer, an effect which is not accounted for in the analytical solution. This effect could be accounted for by also considering the symmetric part of the analytical solution; see Figure 2. For the present case however, this effect is small.

Figure 12 shows the compliance $C_{o h} / C_{b t}$ as a function of the span length, $L$, for two layer thicknesses, $t=.76$ and $3 \mathrm{~mm}$. For short span lengths, the overhang has a significant influence on the compliance. For large span lengths, however, $C_{o h} / C_{b t}$ approaches unity for both layer thicknesses, which shows that ordinary beam theory is recovered.

Figure 13 shows the shear stress distribution, $\tau / \bar{\tau}$, in the layer for three overhang lengths $c=0$, $c=0.127 \mathrm{~m}$, and $c=c_{\infty}(0.506 \mathrm{~m})$. It is seen that an overhang causes substantial changes in the shear stress close to the supports. This will also affect the distribution of the normal force and bending moments 


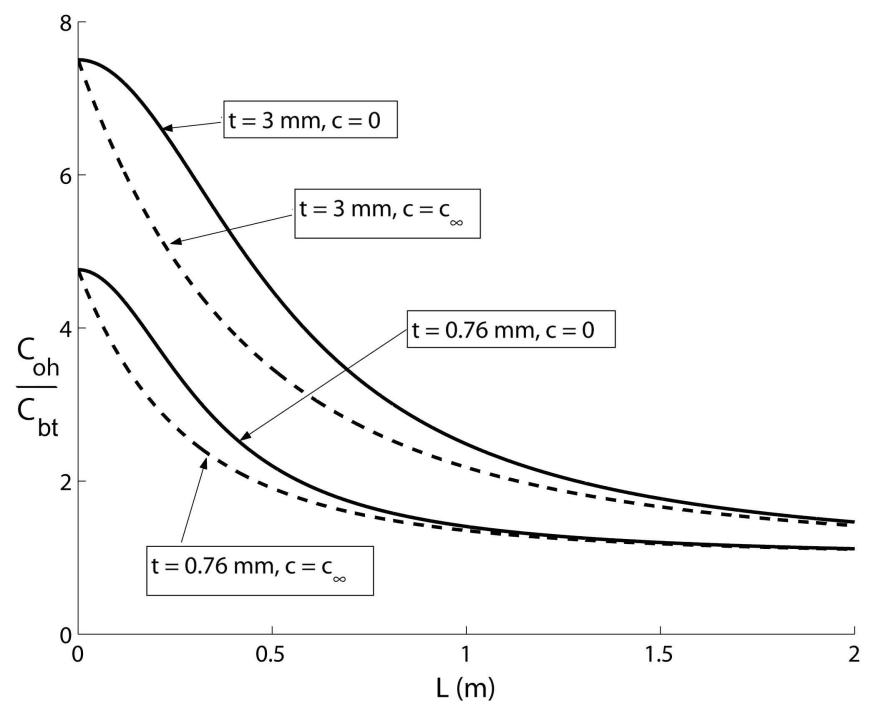

Figure 12. Compliance as a function of span length for beams without overhang (continuous lines) and limiting overhang (dashed lines). $E=70 \mathrm{GPa}, G=3.1 \mathrm{MPa}$, $h=6.35 \mathrm{~mm}, L=0.2 \mathrm{~m}$.

in the adherends and the deflection. For the beam with $c=c_{\infty}$ the shear stress in the layer decays to zero at the left end of the beam. This case represents a limiting value of the overhang. If the overhang is increased beyond this value, the stress distribution (and compliance) will not change.

\section{Experiments}

Rubber testing. The shear modulus, $G$, of the rubber layer was determined using the following equation derived from the statistical theory of rubber elasticity [Aklonis and MacKnight 1983],

$$
\sigma=G\left(\lambda-\frac{1}{\lambda^{2}}\right)
$$

where incompressibility has been assumed, and $\sigma$ represents the tensile stress (calculated based on the original cross sectional area), and $\lambda$ is the extension ratio of the rubber $\left(\lambda=l / l_{0}\right.$, where $l$ and $l_{0}$ are the deformed and undeformed lengths of the rubber specimen). By constructing plots of $\sigma$ versus $\lambda-\lambda^{-2}$ the shear modulus, $G$, is the slope of the initially linear portion of the curve.

A rectangular specimen of $2.54 \mathrm{~cm}$ width and $3 \mathrm{~mm}$ thickness was cut from a flat EPDM rubber sheet. The tensile tests were conducted on specimens using a gage length of $30.5 \mathrm{~cm}$ at a crosshead rate of $2.5 \mathrm{~mm} / \mathrm{min}$. The extension ratio, $\lambda$, was calculated from the crosshead displacement. The rubber shear modulus was determined to be $G=3.11 \pm .07 \mathrm{MPa}$.

Flexure testing. Three-point flexure tests were conducted on beams with aluminum adherends connected by a rubber layer. The adherends were $6.53 \mathrm{~mm}$ thick and $51 \mathrm{~mm}$ wide, with a $3.0 \mathrm{~mm}$ thick rubber layer. 


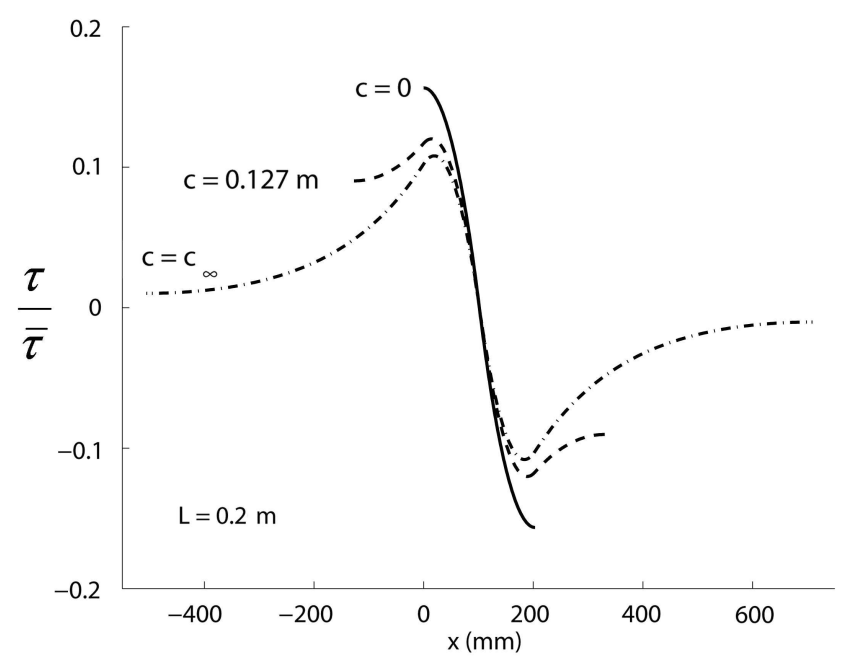

Figure 13. Shear stress distribution for beams with overhang lengths $c=0, .127 \mathrm{~m}$ and $c_{\infty} . E=70 \mathrm{GPa}, G=3.1 \mathrm{MPa}, L=.2 \mathrm{~m}, h=6.35 \mathrm{~mm}, t=3 \mathrm{~mm}$. The shear stress is normalized by $\bar{\tau}$ given by Equation (10).

A critical test of the analytical model is to determine if the effect of the overhang on compliance is borne out experimentally. For that purpose the experiments were conducted at a constant span length, $L=20.3 \mathrm{~cm}$, and at overhang lengths $c=2.54,5.08,7.62,10.2$, and $12.7 \mathrm{~cm}$.

The bonding surfaces of the aluminum beams were grit blasted and treated with silane for adhesive bonding to an EPDM rubber layer. The rubber layer was abraded and treated with a primer before bonding to the adherends using a two-component epoxy adhesive (Applied Poleramic SC-11). Thin scrim cloth layers were inserted between the rubber layer and the adherends to maintain a uniform bond line.

The beams were tested in an Instron 4484 load frame in a three-point flexure fixture with $19.1 \mathrm{~mm}$ diameter cylindrical rollers at the supports and load introduction locations. Figure 14 shows a photo of the actual test setup. To achieve a more uniform load introduction, $1.6 \mathrm{~mm}$ thick rubber pads were inserted between the loading and support rollers and the beam. The displacement of the beams was measured on the top surface above each support, and on the bottom surface under the central load introduction line using LVDTs. The accuracy of the LVDTs was $\pm .0025 \mathrm{~mm}$, or better. The actual deflection of the lower beam surface at the center span $(x=L / 2)$ was determined by subtracting the average displacement at the supports from that at the central load introduction. By this procedure, it was possible to compensate for displacement contributions from the load cell and fixture as well as local deformations in the support regions. By measuring the displacement of the lower surface under the central load, local load introduction effects were eliminated.

The flexure tests were conducted at a cross head rate of $2.5 \mathrm{~mm} / \mathrm{min}$. The beam compliance was determined from the corrected central beam deflection $(\delta)$ divided by the applied load $(P), C=\delta / P$.

In order to satisfy the desired range of overhang lengths at a constant span length $(L=20.3 \mathrm{~cm})$ without introducing multiple beam specimens, the initially $45.7 \mathrm{~cm}$ long beam was loaded to very small displacements in order to maintain linear elastic reversible response. Following this test, the beam was 


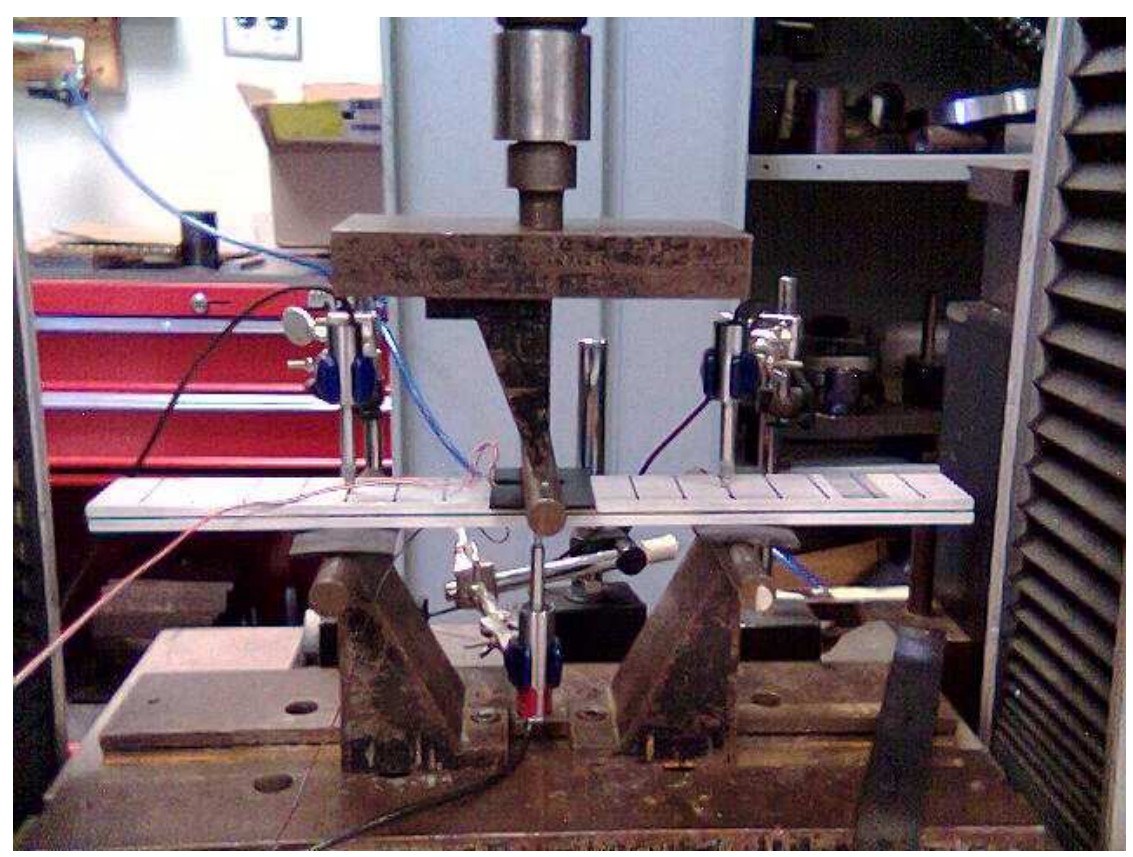

Figure 14. Experimental setup for compliance determination of three-point flexure loaded beams with rubber interlayer.

unloaded, removed from the fixture, and a $2.54 \mathrm{~cm}$ long section of each end was cut off in order to achieve a beam with a shorter overhang length. This procedure was repeated until the shortest $(2.54 \mathrm{~cm})$ overhang length was accomplished.

Figure 15 shows compliance of the beam versus overhang length. The solid circles represent experimental test data determined as described above, while the continuous curve represents predictions from Equation (28), with $E=70 \mathrm{GPa}$ and $G=3.1 \mathrm{MPa}$. It is verified that the presence of an overhang reduces the compliance, and the test data are in very good agreement with the analytical predictions, lending confidence to the approach adopted in this study.

The squares in Figure 15 represent FE results according to the model used in connection with Figure 11. The comparison to the analytical solution is very good. Thus, the predicted reduction of compliance with increasing overhang length, $c$, is confirmed by the FE results.

\section{Concluding remarks}

The beam analysis presented here shows that beams with a flexible interlayer behave quite differently from ordinary homogeneous beams in the sense that a highly localized shear deformation substantially adds to the flexibility and decoupling characteristics of the structure. These effects are quantified through an analytical form of the compliance for the three-point flexure geometry. It is also shown and quantified how an overhang portion of the beam reduces the beam compliance. Conditions for full coupling and full decoupling are formulated for beams with thin interlayers, and expressed in the form of a dimensionless decoupling parameter. The analysis is intended as a design tool that is simpler to use and more 


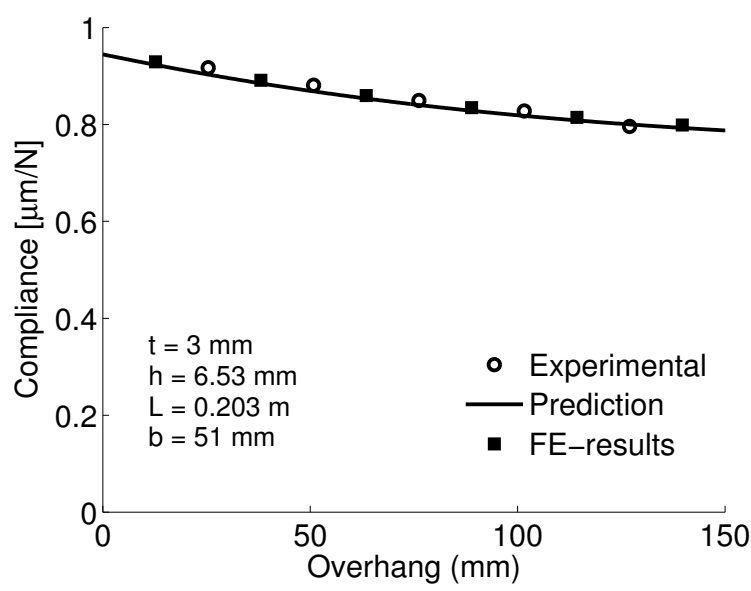

Figure 15. Compliance as a function of overhang length. $E=70 \mathrm{GPa}, G=3.1 \mathrm{MPa}$ $h=6.53 \mathrm{~mm}, b=51 \mathrm{~mm}, t=3 \mathrm{~mm}$, and $L=.203 \mathrm{~m}$. Open circles represent experimental data and the continuous line predictions using Equation (28). Solid squares represent results from FE analysis with Poisson's ratio $v=0.4999$ for the rubber and $v=0.33$ for the aluminum.

computationally efficient than finite elements. Comparison of the analytical model to a continuum finite element model shows excellent agreement. Experiments on three-point flexure loaded aluminum beams with a rubber interlayer were in favorable agreement with the beam analysis predictions.

\section{Acknowledgements}

The authors would like to acknowledge Dr. John Tierney for his valuable contributions.

\section{References}

[Aklonis and MacKnight 1983] J. J. Aklonis and W. J. MacKnight, Introduction to polymer viscoelasticity, 2nd ed., Wiley, New York, 1983.

[Alfredsson 2004] K. S. Alfredsson, "On the instantaneous energy release rate of the end-notch flexure adhesive joint specimen”, Int. J. Solids Struct. 41:16-17 (2004), 4787-4807.

[Bogetti et al. 1988] T. A. Bogetti, J. W. Gillespie Jr., and R. B. Pipes, "Evaluation of the IITRI compression test method for stiffness and strength determination", Compos. Sci. Technol. 32:1 (1988), 57-76.

[Choi and Horgan 1977] I. Choi and C. O. Horgan, "Saint-Venant's principle and end effects in anisotropic elasticity", J. Appl. Mech. (Trans. ASME) 44 (1977), 424-430.

[Davila and Chen 2000] C. G. Davila and T.-K. Chen, "Advanced modeling strategies for the analysis of tile-reinforced composite armor”, Appl. Compos. Mater. 7:1 (2000), 51-68.

[Fischer et al. 2006] C. Fischer, S. A. Braun, P.-E. Bourban, V. Michaud, C. J. G. Plummer, and J.-A. E. Manson, "Dynamic properties of sandwich structures with integrated shear-thickening fluids", Smart Mater. Struct. 15:5 (2006), 1467-1475.

[Frostig and Baruch 1990] Y. Frostig and M. Baruch, "Bending of sandwich beams with transversely flexible core", AIAA J. 28:3 (1990), 523-531.

[Gere and Timoshenko 1984] J. M. Gere and S. P. Timoshenko, Mechanics of materials, 2nd ed., Brookes/Cole Engineering Division, Monterey, CA, 1984. 
[Horgan 1972] C. O. Horgan, “On Saint-Venant's principle in plane anisotropic elasticity”, J. Elasticity 2:3 (1972), 169-180.

[Horgan and Knowles 1983] C. O. Horgan and J. K. Knowles, "Recent developments concerning Saint-Venant's principle", Adv. Appl. Mech. 23 (1983), 179-269.

[Mahdi and Gillespie 2004] S. Mahdi and J. W. Gillespie, Jr., "Finite element analysis of tile-reinforced composite structural armor subjected to bending loads", Compos. Part B Eng. 35:1 (2004), 57-71.

[Mahdi et al. 2003] S. Mahdi, B. A. Gama, S. Yarlagadda, and J. W. Gillespie Jr., "Effect of the manufacturing process on the interfacial properties and structural performance of multi-functional composite structures", Compos. Part A Appl. S. 34:7 (2003), 635-647.

[Mechanica 2007] Mechanica, "Pro/MECHANICA, Mechanica Wildfire 3.0”, 2007, Available at http://www.ptc.com/partners/ hardware/current/prom.htm.

[Reissner 1947] E. Reissner, “On bending of elastic plates”, Q. Appl. Math. 5 (1947), 55-68.

[Reissner 1948] E. Reissner, "Finite deflections of sandwich plates", J. Aeronautical Sci. 15 (1948), 435-440.

[Timoshenko and Woinowsky-Kreiger 1959] S. Timoshenko and S. Woinowsky-Kreiger, Theory of plates and shells, 2nd ed., McGraw-Hill, New York, 1959.

[Whitney 1973] J. M. Whitney, "Shear correction factors for orthotropic laminates under static load", J. Appl. Mech. (Trans. ASME) 40 (1973), 302-304.

[Zenkert 1997] D. Zenkert, An introduction to sandwich construction, EMAS, London, 1997.

Received 24 Oct 2006. Accepted 7 May 2007.

K. S. ALFREDSSON: svante.alfredsson@his.se

University of Skövde, S-541 28 Skövde, Sweden

T. A. BogetTi: bogetti@arl.army.mil

Army Research Laboratory, Aberdeen Proving Ground, Aberdeen, MD 21005, United States

L. A. CARLSSON: carlsson@fau.edu

Department of Mechanical Engineering, Florida Atlantic University, Boca Raton, FL 33431, United States

J. W. GILlESPIE, JR.: gillespie@udel.edu

Center for Composite Materials, University of Delaware, Newark, DE 19716, United States

A. YIOURNAS: yiournas@udel.edu

Dynamic Science Inc. DTSD, Aberdeen, MD 21001, United States 